\title{
INSECT GALLS FROM SERRA DE SÃO JOSÉ (TIRADENTES, MG, BRAZIL)
}

\author{
MAIA, V. C. ${ }^{1}$ and FERNANDES, G. W. ${ }^{2}$ \\ ${ }^{1}$ Departamento de Entomologia, Museu Nacional, Quinta da Boa Vista, São Cristóvão, \\ CEP 209940-040, Rio de Janeiro, RJ, Brazil \\ ${ }^{2}$ Ecologia Evolutiva de Herbívoros Tropicais, DBG, ICB, Universidade Federal de Minas Gerais, \\ C.P. 486, CEP 30161-970, Belo Horizonte, MG, Brazil \\ Correspondence to: Valéria Cid Maia, Museu Nacional, Quinta da Boa Vista, São Cristóvão, CEP 209940-040, \\ Rio de Janeiro, RJ, Brazil, e-mail: maiavcid@acd.ufrj.br \\ Received February 21, 2003 - Accepted July 30, 2003 - Distributed August 31, 2004
}

(With 107 figures)

\begin{abstract}
One hundred thirty-seven morphotypes of insect galls were found on 73 plant species (47 genera and 30 families) in Serra de São José, in Tiradentes, MG, Brazil. Fabaceae, Myrtaceae, Asteraceae, and Melastomataceae were the plant families that supported most of the galls ( $49.6 \%$ of the total). Galls were mostly found on leaves and stems (66.4\% and 25.5\%, respectively). Galls were induced by Diptera, Lepidoptera, Coleoptera, Hemiptera (Sternorrhyncha), Hymenoptera, and Thysanoptera. The majority of them (73.7\%) were induced by gall midges (Cecidomyiidae: Diptera). Besides the gall inducers, other insects found associated with the galls were parasitoids (Hymenoptera), inquilines (Coleoptera, Lepidoptera, Diptera, and Hemiptera), and predators (Diptera).
\end{abstract}

Key words: galls, insects, rupestrian fields, cerrado, Minas Gerais.

\section{RESUMO}

\section{Galhas de insetos da Serra de São José (Tiradentes, MG, Brasil)}

Foram encontrados 137 tipos de galhas de insetos em 73 espécies de plantas (47 gêneros e 30 famílias) na Serra de São José (Tiradentes, MG, Brasil). Fabaceae, Myrtaceae, Asteraceae e Melastomataceae foram as famílias de plantas com maior diversidade de galhas (49,6\% do total). As galhas predominaram nas folhas e caules $(66,4 \%$ e $25,5 \%$, respectivamente). Como galhadores, encontramos espécies de Diptera, Lepidoptera, Coleoptera, Hemiptera (Sternorrhyncha), Hymenoptera e Thysanoptera. A maioria das galhas $(73,7 \%)$ foi induzida por Cecidomyiidae (Diptera). Além dos galhadores, outros insetos foram encontrados associados às galhas, como parasitóides (micro-Hymenoptera), inquilinos (Coleoptera, Lepidoptera, Diptera e Hemiptera) e predadores (Diptera).

Palavras-chave: galhas, insetos, campos rupestres, cerrado, Minas Gerais.

\section{INTRODUCTION}

Galls are abnormal plant growths that occur in response to feeding or other stimuli by foreign organisms (viruses, bacteria, fungi, mites, insects, and others). Insects are the most frequent gall-inducers, especially gall midges (Diptera, Cecidomyiidae). Some Hemiptera (Psyllidae,
Agaontidae, Aphidae, Adelgidae, Pemphigidae), Hymenoptera (Tenthredinidea, Cynipidae, Eurytomidae), Coleoptera (Curculionidae, Cerambycidae), Lepidoptera (Momphidae, Heliozelidae, Tortricidae), Thysanoptera, and other Diptera (Tephritidae, Agromyzidae, Chloropidae, Fergusoninidae) are also known to induce galls on a vast number of host plants. 
Galling insects are most species-rich in the rupestrian fields and in the cerrado (savanna) vegetation of neotropical southeastern Brazil (Fernandes \& Price, 1988; Lara \& Fernandes, 1996). Despite their richness, little is known about the taxonomy of these galling species.

We surveyed the Serra de São José, an area of rupestrian fields and cerrado in the State of Minas Gerais, southeastern Brazil (21 $03-07^{\prime} \mathrm{S}$ and $44^{\circ} 06-$ 13 'W) in an attempt to broaden our knowledge of galling species diversity in the Brazilian cerrado. The serra is a $15 \mathrm{~km}$ long mountain range, 900 to $1,430 \mathrm{~m}$ above sea level, running from WSW to ENE at the contact zone of two main mountain chains: the Espinhaço Range which extends northward and the Serra da Mantiqueira running from south and west (Alves, 1992). The local climate is classified as Cwa (continental with dry winter). Absolute temperatures oscillate greatly, thus constituting a stress factor for plant species (Alves, op . cit.).

Serra flora comprises about 800 species, and the best represented vascular plant families are the Orchidaceae, Asteraceae, Poaceae, and Melastomataceae. Some species, namely Sarcoglottis caudata, Liparis beckeri, Palexia phallocallosa, Eugenia langsdorfii, and Croton gnidiaceus are presently considered narrow endemics of the São José Range (Alves, 1992).

\section{MATERIAL AND METHODS}

The Serra of São José was investigated for galls over a period of 12 months from August 2001 to July 2002. Collections were made monthly along three pathways: the access roads to "Cachoeira do Mangue" (hereafter CM), "Calçada da Serra" (hereafter CS), and "Cachoeira do Bom Despacho" (hereafter BD) (Fig. 1). The vegetation was examined along each pathway for ca. 8 hours per visit. All plant organs were investigated, except for the roots. Samples of host plants, preferably in the fertile state, were pressed in the field. The Asteraceae species were identified by Roberto L. Steves (Universidade do Estado do Rio de Janeiro, Brazil) and all other plant species by Ruy J. Válka Alves (Museu Nacional, Rio de Janeiro, hereafter MNRJ, Brazil). Voucher specimens were included in the Herbarium (R) whenever fertile plant material was collected. Authors and scientific plant names were checked on "mobot.mobot.org".

The galls were photographed and the negatives organized into an archive. Samples of dried galls were incorporated in the Diptera collection of MNRJ.

Immature insects were obtained by from the dissection of each kind of gall under a stereoscopic microscope. This procedure also enabled insect habit determination (whether of inquilines, predators, parasitoids, or galling species).

Pupal exuviae and adults were obtained by keeping samples of each gall individually in plastic pots layered at the bottom with damp cotton and covered by fine screening. Galls of the species whose larvae pupate in the soil were kept in pots with a layer of soil on the botton. All pots were checked daily.

Larvae, pupae, pupal exuviae, and adults of Cecidomyiidae were preserved in $70 \%$ alcohol and later mounted on slides, following the methodology of Gagné (1994). The gall midge genera were identified based mainly on the keys of Gagné $(1989,1994)$. The other insects (Hymenoptera, Coleoptera, Lepidoptera, and Diptera: Chloropidae) were identified by Maria Antonieta Pereira de Azevedo (MNRJ), Sérgio Vanin (Universidade de São Paulo, Brazil), Victor Becker (Brazil), and Luc De Bruyn (Institute of Nature Conservation, Belgium), respectively.

All material is deposited in the collections of MNRJ, except part of the Coleoptera, Chloropidae (Diptera), and Lepidoptera samples which were donated to S. Vanin, L. De Bruyn, and V. Becker, respectively.

\section{RESULTS}

One hundred thirty-seven (137) morphotypes of insect galls were found in the Serra de São José. They were induced by species of Diptera (Cecidomyiidae, Tephritidae, and Muscomorpha), Hemiptera (Sternorrhyncha), Lepidoptera, Coleoptera, Thysanoptera, and Hymenoptera (Table 1). The Cecidomyiidae were the most common gall inducers, being responsible for $73.7 \%$ of the recorded galls. The Lepidoptera was second, with only $7.3 \%$. 
TABLE 1

Abundance of galling insect taxa in the Serra de São José (Tiradentes, MG, Brazil).

\begin{tabular}{|l|c|}
\hline \multicolumn{1}{|c|}{ Galling species } & N. of gall morphotypes \\
\hline Cecidomyiidae (Diptera) & 101 \\
\hline Lepidoptera & 10 \\
\hline Hemiptera (Sternorrhyncha) & 8 \\
\hline Curculionidae (Coleoptera) & 2 \\
\hline Eulophidae (Hymenoptera) & 2 \\
\hline Thysanoptera & 2 \\
\hline Tephritidae (Diptera) & 1 \\
\hline Muscomorpha (Diptera) & 1 \\
\hline Undetermined insect & 10 \\
\hline
\end{tabular}

The galling species were associated with 73 species of plants belonging to 47 genera and 30 families. Fabaceae, Myrtaceae, Asteraceae, and Melastomataceae were the plant families with the greatest gall richness of galls, with 20,18, 16, and 14 kinds of gall, respectively. These four families comprised about $49.6 \%(\mathrm{~N}=68)$ of all galls (Table 2). Galls induced by Cecidomyiidae were frequent on almost all plant families. Only in the Melastomataceae the trend was toward the induction by lepidopterans $(50 \%)$.

The plant species which supported the higher number of galls were Protium heptaphyllum (Burseraceae, $\mathrm{N}=7$ ), Copaifera langsdorffii (Fabaceae, $\mathrm{N}=6$ ), Myrcia sp. (Myrtaceae, $\mathrm{N}=6$ ), Croton floribundus (Euphorbiaceae, $\mathrm{N}=5$ ), and Miconia theaezans (Melastomataceae, $\mathrm{N}=5$ ). Although Asteraceae was one of the plant families with the greatest richness of galls, each species supported only 1 or 2 gall morphotypes. Contrasting with the Asteraceae, the species belonging to Fabaceae and Myrtaceae species showed a range of from 1 to 6 galls. Melastomataceae presented a similar pattern: from 1 to 5 gall morphotypes.

Some host plants are considered rare in the State of Minas Gerais, probably due to indiscriminate human exploitation of their fruits, seed, or wood (e.g., Anona crassiflora (Annonaceae, "araticum"), Astronium fraxinifolium (Anacardiaceae, "gonçaleira"), Byrsonima verbascifolia (Malpighiaceae, "murici-deflor-amarela"), Campomanesia pubescens (Myrtaceae, "gabiroba"), and Stryphnodendron adstringens (Fabaceae, "barbatimão") (Brandão et al., 1992).
Most galls were induced on leaves (66.4\%). The stem was the second most attacked plant organ ( $25.5 \%)$, followed by buds (13.1\%), petiole $(2.2 \%)$, and flower bud or inflorescence (1.4\%). None of the studied galls occurred on fruits; roots were not investigated. The majority of the gall inducers attacked a specific plant part, but some of them $(\mathrm{N}=9)$ attacked simultaneously two or three plant parts (Table 3).

In addition to the gall formers, other insects were also found in the galls. These were classified as parasitoids, inquilines, or predators.

Friebrigella sp. (Chloropidae: Diptera), the only species of Chloropidae in our study, was found every month in galls of Lepidoptera on Leandra aurea, a genus including only predator species. In the same gall, gall midge larvae were found as inquilines between gall hairs. Muscomorpha larvae (2 spp.), Coleoptera (4 spp.), and Lepidoptera (13 spp.) were found in 18 gall morphotypes, as galling species (1, 2 , and 10 spp., respectively) or as inquilines (1,2, and 3 spp., respectively). Sciaridae (2 spp.), Psyllidae (1 sp.), and Heteroptera (1 sp.) were found in four different galls only as inquilines. Hymenoptera species were found in about $35 \%$ of the gall morphotypes; none of them presented inquilinous habits. Almost all were parasitoids, except two species which were considered gall inducers (Table 4). Among the Hymenoptera, we found species belonging to the Eurytomidae, Braconidae, Platygastridae, Eulophidae, Pteromalidae, Ichneumonidae, and Encyrtidae. The two hymenopteran galling species belong to the Eulophidae (Table 5). 
One hundred four (104) species of Cecidomyiidae were obtained, most of them as galling species $(\mathrm{N}=101,97.1 \%)$, but also as inquilinous $(\mathrm{N}=2,1.94 \%)$ and as predators $(\mathrm{N}=$ 1, 0.96\%) (Tab. 4).
The majority of the gall midges (Cecidomyiidae) from Serra de São José showed only one generation per year and almost all of the galls were rare. Hence, few samples were obtained and many gall midges were, thus, not determined.

TABLE 2

Richness of insect galls per plant family in the Serra de São José (Tiradentes, MG, Brazil).

\begin{tabular}{|c|c|}
\hline Plant family & N. of gall morphotypes \\
\hline Anacardiaceae & 2 \\
\hline Annonaceae & 0 \\
\hline Asteraceae & 16 \\
\hline Boraginaceae & 1 \\
\hline Burseraceae & 7 \\
\hline Clusiaceae & 4 \\
\hline Chrysobalanaceae & 1 \\
\hline Dilleniaceae & 2 \\
\hline Erythroxylaceae & 5 \\
\hline Euphorbiaceae & 9 \\
\hline Fabaceae & 20 \\
\hline Lamiaceae & 2 \\
\hline Lauraceae & 1 \\
\hline Lythraceae & 1 \\
\hline Malpighiaceae & 4 \\
\hline Melastomataceae & 14 \\
\hline Myrsinaceae & 2 \\
\hline Myrtaceae & 18 \\
\hline Nyctaginaceae & 3 \\
\hline Piperaceae & 5 \\
\hline Rubiaceae & 3 \\
\hline Sapindaceae & 3 \\
\hline Scrophulariaceae & 1 \\
\hline Smilacaceae & 1 \\
\hline Tiliaceae & 3 \\
\hline Ulmaceae & 3 \\
\hline Umbelliferae & 1 \\
\hline Verbenaceae & 2 \\
\hline Vochysiaceae & 1 \\
\hline Winteraceae & 1 \\
\hline
\end{tabular}


TABLE 3

Abundance of insect galls on plant organs in the Serra de São José (Tiradentes, MG, Brazil). The parenthesized values indicate the number of gall morphotypes that occurred simultaneously on other plant organs.

\begin{tabular}{|l|c|}
\hline \multicolumn{1}{|c|}{ Plant part } & N. gall morphotypes \\
\hline Leaves & $91(9)$ \\
\hline Stem & $36(9)$ \\
\hline Bud & $18(1)$ \\
\hline Petiole & $3(3)$ \\
\hline Flower bud or inflorescence & 2 \\
\hline Petiole, veins (leaves) and stem & 1 \\
\hline Petiole and midvein (leaves) & 2 \\
\hline Stem and leaves & 7 \\
\hline Bud and leaves & 1 \\
\hline
\end{tabular}

TABLE 4

Habit frequency of insects associated with galls in the Serra de São José (Tiradentes, MG, Brazil).

\begin{tabular}{|l|c|c|c|c|c|}
\hline \multirow{2}{*}{ Insect taxon } & \multicolumn{5}{|c|}{ N. species as } \\
\cline { 2 - 6 } & Gall inducer & Inquiline & Predator & Parasitoid & Total \\
\hline Cecidomyiidae & 101 & 2 & 1 & - & 104 \\
\hline Hymenoptera & 2 & - & - & 46 & 48 \\
\hline Lepidoptera & 10 & 3 & - & - & 13 \\
\hline Coleoptera & 2 & 2 & - & - & 4 \\
\hline Sciaridae & - & 1 & - & - & 2 \\
\hline Muscomorpha & 1 & 1 & - & - & 2 \\
\hline Thysanoptera & 2 & - & - & - & 1 \\
\hline Psyllidae & - & 1 & - & - & 1 \\
\hline Tephritidae & 1 & - & - & - & 1 \\
\hline Chloropidae & - & - & 1 & - & 2 \\
\hline Heteroptera & - & 1 & - & - & 1 \\
\hline
\end{tabular}

Nevertheless, 10 genera of Cecidomyiidae are recorded, namely: Asphondylia Loew, 1850; Clinodiplosis Kieffer, 1895; Contarinia Rondani, 1860; Dactylodiplosis Rübsaamen, 1916; Dasineura Rondani, 1840; Lopesia Rübsaamen, 1908; Myrciariamyia Maia, 1994; Neolasioptera Felt, 1908; Stephomyia Tavares, 1916; and Zalepidota Rübsaamen, 1907. Among them, the most common genus was Neolasioptera with 6 species (galling species). Asphondylia, Contarinia, Dasineura, Neolasioptera, and Stephomyia species had already been recorded for the State of Minas Gerais (Fernandes et al., 1988; Maia et al., 2002), but these are the first records of Clinodiplosis, Dactylodiplosis, Lopesia, and Myrciariamyia species for this state. As no previous gall survey was done in Serra de São José, all records are new for this locality. 
This is also the first record of insect galls on the following 22 plant species: Anona crassiflora Mart, Aspilia duarteana Santos, Baccharis microcephala (Less.) DC., Baccharis reticularia DC., Mikania lindbergii Baker, Mikania micrantha Kunth., Mikania sessilifolia DC., Vannilosmopsis erythropapa Schult, Vernonia crotonoides Schult. Bip., Vernonia obscura Less., Davilla braziliana DC., Croton gnidiaceus Baill., Croton timandroides (Didr.) Müll. Arg., Stryphnodendrum adstringes (Aubl.) Benth, Byrsonima variabilis A. Juss., Leandra aurea (Cham.) Cogn., Miconia theaezans (Bonpl.) Cogn., Tibouchina candolleana (DC.) Cogn., Rapanea andina Mez., Myrciaria tenella (DC.) O. Berg., Palicourea rigida Kunth, and Buchnera rosea Kunth.

Data on insect galls are presented here under host plant species in alphabetical order and include their description, galling species identification, other associated insects, period of gall occurrence, site of collection (CS, CM, and BD), and deposited material (galls).

\section{Anacardiaceae}

Astronium fraxinifolium Schott ex Spreng: Opened leaf gall. Galling species: Hemiptera (Sternorrhyncha). Period: August (CS). Comments: gall recorded on Astronium sp. for the restinga of Barra de Maricá (Maricá, RJ) by Maia (2001, p. 593, Fig. 2).

Schinus terebinthifolius Raddi: Opened leaf gall. Galling species: Coccidea (Hemiptera, Sternorrhyncha). Period: March (CM). Deposited material: 22 galls. Comments: similar to gall described by Houard, 1933 (p. 204-211; Fig. Q).

\section{Annonaceae}

Anona crassiflora Mart.: Spheroid leaf gall (Fig.

2). Galling species: Hemiptera (Sternorrhyncha). Other associated insects: parasitoids - Eurytomidae (1 female), Braconidae (1 female). Period: May (CM), September (CM). Deposited material: 13 galls (03.V.2001). Comments: Houard (1933) listed 4 kinds of galls on Anona sp. (3 induced by Cecidomyiidae, Diptera and one induced by Psyllidae (Hemiptera); none of them similar in shape. This is the first record of gall on Anona crassiflora.

\section{Asteraceae}

Aspilia duarteana Santos: Globoid stem swelling (Fig. 3). Galling species: Cecidomyiidae. Period: March (CS). Deposited material: 5 galls (03.V.2001). Comments: first record of gall on Aspilia duarteana.

Baccharis microcephala (Less.) DC: Ovoid bud gall (Fig. 4). Galling species: Cecidomyiidae. Other associated insects: parasitoids - Eulophidae sp. (2 females); Galeopsomyia sp. (Eulophidae, 2 males, 1 female); Eurytoma (Eurytomidae, 1 male). Period: April (CM); May (CS and CM). Deposited material: 4 galls, 25.IV.2002.

Midvein swelling (Fig. 5). Galling species: Cecidomyiidae. Other associated insects: Eulophidae sp. 1 (1 male and 1 female), Eulophidae sp. 2 (2 females). Period: September-October, (CS and CM); January (CS); April (CM); May (CS and CM). Deposited material: 4 galls, 24.X.2001.

Comments: first records of gall on Baccharis microcephala.

Baccharis reticularia DC: Swelling of petiole, veins or stem, greenish or reddish (Fig. 6). Galling species: Cecidomyiidae. Other associated insects: parasitoids - Platygastridae ( 3 females and 1 male). Period: March (BD); October (CM); November (CM and CS); December (CS and CM); April (CM); May (CM). Deposited material: 6 galls on stem (2 galls, 28.IX.2001; 2 galls, 25.X.2001; 1 gall, 20.XI.2001; 1 gall, 28.V.2002); 2 galls on petiole (25.X.2001), 3 on midvein (25.X.2001).

Marginal leaf roll, reddish (Fig. 7). Galling species: Lasiopteridi (Cecidomyiidae). Other associated insects: Heteroptera (3 nimphs). Period: October (CM); May (CM). Deposited material: 11 galls (6 galls, 25.X.2001; 5 galls, 20.XI.2001).

Cylindrical outgrowths of the leaves (Fig. 8). Galling species: Cecidomyiidae. Period: May (CM).

Comments: first records of gall on Baccharis reticularia.

Baccharis serrulata (Lam.): Pres. Spherical red leaf or stem gall (Fig. 9). Galling species: Cecidomyiidae. Other associated insects: parasitoids Hymenoptera. Period: November (CS). Deposited material: 1 gall, 21.XI.2001. Comments: Fernandes et al. (1996) listed 121 kinds of insect galls on Baccharis spp., including B. serrulata. 
TABLE 5

Distribution of Hymenoptera on host plants and their association with galling species in the Serra de São José (Tiradentes, MG, Brazil).

\begin{tabular}{|c|c|c|c|}
\hline Host plant & Plant part & Galling species & Parasitoid \\
\hline Anona crassifolia & leaf & Sternorrhyncha & Eurytomidae/Braconidae \\
\hline Baccharis microcephala & leaf (midvein) & Cecidomyiidae & Eulophidae/Eurytomidae \\
\hline \multirow[t]{2}{*}{ Baccharis reticularia } & petiole/vein/stem & Cecidomyiidae & Platygastridae \\
\hline & bud & Cecidomyiidae & $\begin{array}{l}\text { Eulophidae/ } \\
\text { Galeopsomyia (Eulophidae)/ } \\
\text { Eurytoma (Eurytomidae) }\end{array}$ \\
\hline Baccharis serrulata & leaf & Cecidomyiidae & Hymenoptera \\
\hline Mikania lindbergii & stem & \multicolumn{2}{|c|}{ Neolasioptera sp. (Cecidomyiidae) Hymenoptera } \\
\hline V. eryhtropapa & leaf & \multicolumn{2}{|c|}{ Asphondylia sp.n. (Cecidomyiidae)Hymenoptera } \\
\hline Vernonia polyanthes & bud & T. rudolphi (Tephritidae) & Hymenoptera \\
\hline \multirow[t]{4}{*}{ Calophyllum sp. } & leaf & Lopesia sp. (Cecidomyiidae) & Galeopsomyia/Pteromalidae \\
\hline & & & Eulophidae/Eurytomidae \\
\hline & stem & Lopesia sp. (Cecidomyiidae) & Hymenoptera \\
\hline & leaf & probably Thysanoptera & Hymenoptera \\
\hline Davilla braziliana & leaf & Asphondylia sp. (Cecidomyiidae) & Hymenoptera \\
\hline Erythroxyllum suberosum & leaf & Dasineura sp. (Cecidomyiidae) & Hymenoptera \\
\hline E. frangulifolium & bud & Eulophidae (Hymenoptera) & |------------------ \\
\hline \multirow[t]{4}{*}{ Croton floribundus } & leaf & Clinodiplosis sp. (Cecidomyiidae) & Hymenoptera \\
\hline & leaf & Cecidomyiidae & Hymenoptera \\
\hline & leaf (midvein) & Cecidomyiidae (gen.n.) & Hymenoptera \\
\hline & leaf (roll) & undetermined & Hymenoptera \\
\hline \multirow[t]{2}{*}{ Andira sp. } & stem & Cecidomyiidae & Eulophidae \\
\hline & leaf & Cecidomyiidae & Hymenoptera \\
\hline Copaifera langsdorffii & leaf (discoid) & Cecidomyiidae & Hymenoptera \\
\hline Inga sp. & leaf (midvein) & Cecidomyiidae & Hymenoptera \\
\hline \multirow[t]{2}{*}{ Fabaceae undetermined } & bud (horn) & Cecidomyiidae & Hymenoptera \\
\hline & stem & \multicolumn{2}{|c|}{ Neolasioptera sp. (Cecidomyiidae)Hymenoptera } \\
\hline Byrsonima variabilis & stem & undetermined & $\begin{array}{l}\text { Sycophila (Eurytomidae)/ } \\
\text { Eurytoma (Eurytomidae)/ } \\
\text { Eupelmidae/Eulophidae/ } \\
\text { Ichneunomidae/Platygastridae }\end{array}$ \\
\hline \multirow[t]{3}{*}{ Byrsonima verbascifolia } & leaf & Cecidomyiidae & $\begin{array}{l}\text { Eurytoma (Eurytomidae)/ } \\
\text { Eulophidae }\end{array}$ \\
\hline & & & $\begin{array}{l}\text { Dimeromicrus cecidomyiae } \\
\text { (Torymidae) }\end{array}$ \\
\hline & & & Signiphoridae \\
\hline \multirow[t]{2}{*}{ Leandra aurea } & bud & Lepidoptera & Hymenoptera \\
\hline & leaf & Lepidoptera & Hymenoptera \\
\hline Miconia sp. 2 & leaf/stem & Cecidomyiidae & Hymenoptera \\
\hline Miconia theaezans & bud (spheroid) & Cecidomyiidae & Hymenoptera \\
\hline Tibouchina candolleana & leaf (vein) & Rochadiplosis (Cecidomyiidae) & Hymenoptera \\
\hline Rapanea andina & leaf & Cecidomyiidae & Hymenoptera \\
\hline Eugenia cf. ovalifolia & leaf & Stephomyia (Cecidomyiidae) & Hymenoptera \\
\hline
\end{tabular}


Table 5 (Continued.)

\begin{tabular}{|llll|}
\hline Host plant & Plant part & Galling species & Parasitoid \\
\hline Eugenia cf. ovalifolia & leaf & Stephomyia (Cecidomyiidae) & Hymenoptera \\
\hline Myrcia sp. & bud (ovoid) & Cecidomyiidae & Hymenoptera \\
\hline & leaf (marginal roll) & Cecidomyiidae & Hymenoptera \\
\hline Myrciaria tenella & leaf (roll) & Thysanoptera & Hymenoptera \\
\hline Myrtaceae (undetermined) & leaf (bivalve) & Myrciariamyia (Cecidomyiidae) & Hymenoptera \\
\hline & leaf (tubular) & undetermined & Hymenoptera \\
\hline Guapira sp. & leaf (fold) & Sternorrhyncha & Hymenoptera \\
\hline & leaf & Lopesiini (Cecidomyiidae) & Hymenoptera \\
\hline Piper sp. 1 & stem & Asphondyliini (Cecidomyiidae) & Hymenoptera \\
\hline Piper sp. 3 & leaf/stem & Asphondyliini (Cecidomyiidae) & Hymenoptera \\
\hline $\begin{array}{l}\text { Borreria } \text { cf. } \\
\text { brachystemonoides }\end{array}$ & leaf (midvein) & Cecidomyiidae & Hymenoptera \\
\hline Paullinia sp. & & & Encyrtidaez \\
\hline Luehea cf. divaricata & stem & Cecidomyiidae & Hymenoptera \\
\hline Lantana lilacina & stem & Cecidomyiidae & Hymenoptera \\
\hline & leaf/stem (mamilif.) & Cecidomyiidae & Neolasioptera sp. (Cecidomyiidae) Hymenoptera \\
\hline Qualea parvifolia & stem & Cecidomyiidae & Hymenoptera \\
\hline Drimys brasiliensi & leaf (tubular) & Cecidomyiidae & Hymenoptera \\
\hline
\end{tabular}

Mikania lindbergii Baker: Suculent stem gall (Fig. 10). Galling species: Neolasioptera sp. (Cecidomyiidae). Other associated insects: parasitoids Hymenoptera. Period: September-November (CM); December (CM and CS); January (CM); April-May (CS and CM). Deposited material: 6 galls (1 gall, 2123.III.2001; 2 galls, 20.X.2001; 3 galls, 01.IX.2001). Comments: first record of gall on Mikania lindbergii. Mikania micrantha Kunth: Globoid leaf gall (Fig. 11). Galling species: Cecidomyiidae. Period: May (CM); April (CM). Deposited material: 5 galls on 03.V.2001. Comments: first record of gall on Mikania micrantha.

Mikania sessilifolia DC.: Reddish circular leaf gall. Galling species: Neolasioptera sp. (Cecidomyiidae). Period: April-May (CM).

Tapered swelling of petiole and midvein, hairy (Fig. 12). Galling species: Cecidomyiidae. Period: April (CM). Comments: Gagné (1994) listed many Cecidomyiidae galls on different species of Mikania and Gagné et al. (2001) described 8 Cecidomyiidae galls on Mikania glomerata in Brazil. These are the first records of gall on Mikania sessilifolia.

Vannilosmopsis erythropapa Schult: Spheroid leaf gall, yellowish or whitish, glabrous (Fig. 13). Galling species: Asphondylia sp. n. (Cecidomyiidae). Other associated insects: Hymenoptera and Tephritidae (4 adults). Period: March (CM); May (CM); August (BD); September (BD); October (BD, $\mathrm{CS}$, and $\mathrm{CM}$ ); November (CM and CS); DecemberJanuary (CM); May (CM). Deposited material: 8 galls, 21-23.III.2001. Comments: Eriophyidae gall listed in Houard (1933, p. 394) and Rübsaamen (1908). First record of insect galls on Vannilosmopsis erythropapa.

Vernonia crotonoides Schult. Bip.: Leaf gall, hairy (Fig. 14). Galling species: Cecidomyiidae. Period: May (CM). Deposited material: 2 galls, 03.V.2001. Comments: first record of insect galls on Vernonia crotonoides. 


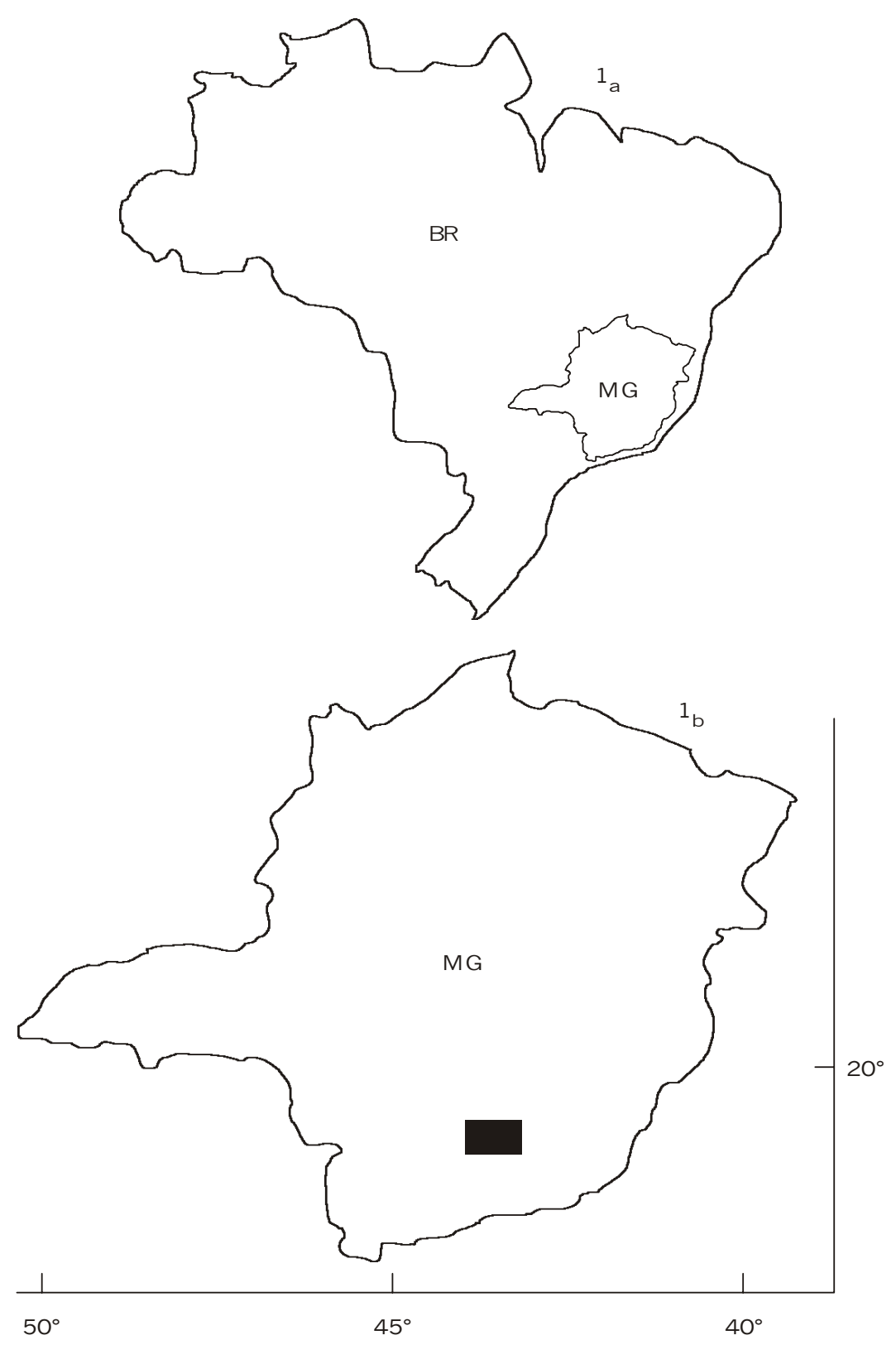

Fig. 1 - Map of Brazil (a) and Minas Gerais State (b). The black rectangle represents the Serra de São José.

Vernonia polyanthes Less: Stem gall, ovoid, brown, glabrous. Galling species: Tomoplagia rudolphi (Diptera, Tephritidae). Other associated insects: Hymenoptera and Tephritidae (1 larva, 30.I.2002). Period: March (CM); January (CM). Deposited material: 3 galls, 03.V.2001. Comments: This gall has already been recorded on the same plant species by Fernandes et al. (1988) for Belo Horizonte (Minas Gerais). Other records of insect galls on Vernonia spp. were made by Gagné, 1994 (gall suculent, thick-walled, monothalamous on stem or petiole on $V$. polyanthes; galling species: Asphondylia sp.; distr.: MG); Möhn, 1964 (tapered stem swelling on Vernonia sp.; galling species: Neolasioptera vernoniensis; distr.: MG); Möhn, 1959 (swollen achene on V. canescens; galling species: Asphondylia herculesi; distr.: El Salvador); Möhn, 1959 and 1960 (swollen bud on V. canescens and V. patans; galling species: Asphondylia ajallai; distr.: El Salvador). 
Vernonia obscura Less: Bud or leaf gall, hairy (Fig. 15). Galling species: Cecidomyiidae. Period: April (CM). Deposited material: 2 galls, 27.V.2002. Comments: first record of gall on this plant.

Vernonia sp.: Stem swelling (Fig. 16). Galling species: Muscomorpha. Period: April (CM). Deposited material: 3 galls, 24.IV.2002. Comments: Houard (1933) listed Lepidoptera and Eriophyidae galls on Vernonia spp. (p. 394).

\section{Boraginaceae}

Cordia cf.: Globoid hairy gall on inflorescence (Fig. 17). Galling species: Cecidomyiidae. Period: September (CS); November-January (CS). Deposited material: 22 galls, 28.IX.2001.

\section{Burseraceae}

Protium heptaphyllum (Aubl.) Marchand: Pouch gall on leaf margin (Fig. 18). Galling species: Cecidomyiidae. Period: September (CM). Deposited material: 2 galls, 01.IX.2001.

Marginal leaf roll (Fig. 19). Galling species: Lopesia sp. (Cecidomyiidae). Period: SeptemberJanuary (CS and CM); April (CS and CM). Deposited material: 4 galls, 28.IX.2001. Comments: Maia (2001) recorded this gall for the restinga of Barra de Maricá (p. 593, Fig. 17).

Opened leaf gall (Fig. 20). Galling species: Hemiptera (Sternorhyncha). Period: October (CM); January (CM).

Midvein swelling. Galling species: Cecidomyiidae. Period: October (CM). Deposited material: 12 galls, 28.IX.2001.

Conical leaf gall parallel to the leaf surface. Galling species: Cecidomyiidae. Period: January (CM).

Conical leaf gall perpendicular to the leaf surface (Fig. 21). Galling species: Dactylodiplosis sp. (Cecidomyiidae). Period: May (CM). Deposited material: 19 galls (15 galls, 27.V.2002; 4 galls, 28.V.2002).

Spherical leaf gall, orangish green, hairy. Galling species: Cecidomyiidae (3 larvae, 28.V.2002). Period: April-May (CM). Deposited material: 27 galls (25.IV.2002).

Comments: Maia (2001) recorded a marginal leaf roll and a conical leaf gall perpendicular to the leaf surface on the same plant species for restinga of Barra de Maricá (Maricá, RJ).

\section{Clusiaceae}

Calophyllum sp.: Elliptical leaf gall, yellow (Fig. 22). Galling species: Lopesia sp. (Cecidomyiidae). Other associated insects: parasitoids - Galeopsomyia sp. (Eulophidae, 5 females, 1 male); Eulophidae sp. (new genus, 1 male); Eurytoma sp. (Eurytomidae, 2 females, 1 male); Pteromalidae sp. (1 female) and inquilinous Sciaridae (2 adults). Period: September-January (CM); April-May (CM). Deposited material: 19 galls, 01.IX.2001.

Stem swelling gall (Fig. 23). Galling species: Lopesia sp. (Cecidomyiidae). Other associated insects: parasitoids - Hymenoptera. Period: September-January (CM); May (CM). Deposited material: 9 galls (6 galls, 01.IX.2001; 1 gall, 20.XI.2001; 2 galls, 28.V.2002).

Marginal leaf roll (Fig. 24). Galling species: probably Thysanoptera. Other associated insects: Hymenoptera and Sciaridae (1 adult). Period: September-December (CM); April (CM).

Linear outgrowth of the leaves (Fig. 25). Galling species: Cecidomyiidae. Period: SeptemberJanuary (CM); April-May (CM). Deposited material: 13 galls (8 galls, 25.X.2001; 5 galls, 25.IV.2002).

Comments: Houard (1933) listed an Eriophyidae gall on Calophyllum calaba Jac.

\section{Chrysobalanaceae}

Licania sp.: Stem swelling. Galling species: Cecidomyiidae. Period: March (CM). Deposited material: 1 gall on 21-23.III.2001. Comments: first record of gall on this plant genus.

\section{Dilleniaceae}

Davilla braziliana DC.: Pine-like bud gall (Fig. 26). Galling species: Asphondylia sp. (Cecidomyiidae). Other associated insects: inquilinous: Clinodiplosis sp. (Cecidomyiidae); Lepidoptera (1 immature specimen). Period: March (CM); August-September (CM); October-November (CM); May (CM). Deposited material: 4 galls (2 galls, 21-23.III.2001 collected by V. C. Maia and 2 galls, 12.X.1999 collected by R. J. V. Alves).

Circular leaf gall (Fig. 27). Galling species: Cecidomyiidae. Other associated insects: parasitoids Hymenoptera. Period: May (CM). Deposited material: 5 galls on 28.V.2002. 
Comments: two kinds of Eriophyidae galls on D. lucida and D. flexuosa (Ref.: Tavares, 1922 and Houard, 1933, p. 241-242); swollen aborted flower on D. rugosa induced by Cecidomyiid (Ref.: Gagné, 1994); leaf blister on D. rugosa induced by Cecidomyiid. Distr. RJ (Ref.: Tavares, 1922). These are the first records of gall on Davilla braziliana.

\section{Erythroxylaceae}

Erythroxylum suberosum St. Hil.: Reddish and hairy leaf gall (Fig. 28). Galling species: Dasineura sp. (Cecidomyiidae). Other associated insects: parasitoids - Hymenoptera. Period: March (CM and BD); September (CM and BD); October (CM); November (BD). Deposited material: 2 galls, 08.IX.1997 collected by R. J. V. Alves. Comments: This gall has already been recorded on the same plant species by Fernandes \& Martins (1985, p. 63, fig. 13). Gagné (1994) listed a spheroid, fuzzy gall induced by Cecidomyiidae on $E$. coelophlebium. Ref.: Fernandes et al. (1988, p. 20, Fig. 7). Distr. MG.

Circular leaf gall (Fig. 29). Galling species: Cecidomyiidae. Period: September-November (BD). Deposited material: 7 galls, 21.XI.2001.

Erythroxylum frangulifolium St. Hilaire: Apical bud gall (Fig. 30). Galling species: Eulophidae (Hymenoptera). Period: May (CM); September (CM); November (BD and CM); December (CM); January (CM); April-May (CM). Deposited material: 1 gall, 03.V.2001. Comments: Fernandes et al. (1988, p. 21, Fig. 22) described the same gall for Belo Horizonte (Minas Gerais).

Marginal leaf roll (Fig. 31). Galling species: Cecidomyiidae. Period: October (CM). Deposited material: 2 galls, 24.X.2001.

Erythroxylum sp.: Blister leaf gall (Fig. 32). Galling species: Cecidomyiidae (mature larvae pupate into soil). Other associated insects: parasitoids Hymenoptera. Period: September (CM); NovemberDecember (CM); April-May (CM). Deposited material: 17 galls, 01.IX.2001.

\section{Euphorbiaceae}

Croton antisyphiliticus Mart.: Circular leaf gall, yellowish (Fig. 33). Galling species: Contarinia sp. (Cecidomyiidae). Period: September (BD); October (BD and CM); November (CM). Deposited material: 3 galls, 29.IX.2001.

Midvein swelling. Galling species: Cecidomyiidae (3 larvae, 25.X.2001). Period: October (BD). Deposited material: 3 galls, 25.X.2001.

Comments: Gonçalves-Alvim \& Fernandes (2001) recorded a stem gall induced by Cecidomyiidae on the same plant species.

Croton floribundus Spreng.: Spheroid hairy gall on leaves or stem (Fig. 34). Galling species: Clinodiplosis sp. (Cecidomyiidae). Other associated insects: parasitoids - Hymenoptera; inquilinous Lepidoptera (2 immature specimens, 30.I.2002; 25.IV.2002). Period: March (CM); August-September (CS); October (CM); December (CS); January (CS); April-May, 2002 (CS). Deposited material: 10 galls (4 galls, 21-23.III.2001; 6 galls, 30.I.2002).

Ovoid leaf gall, slightly hairy (Fig. 35). Galling species: Cecidomyiidae. Other associated insects: parasitoids - Hymenoptera. Period: March (CS); August (CS); November-December (CS); April (CS). Deposited material: 3 galls, 21-23.III.2001.

Discoidal leaf gall (Fig. 36). Galling species: Cecidomyiidae. Period: August-September (CS); April-May (CS). Deposited material: 4 galls, 28.IX.2001.

Midvein swelling (Fig. 37). Galling species: Lasiopteridi, gen. n. (Cecidomyiidae). Other associated insects: parasitoids - Hymenoptera. Period: August (CS); October (CM); November-January (CS). Deposited material: 10 galls, 28.IX.2001.

Marginal leaf roll (Fig. 38). Galling species: undetermined insect. Period: March (CS). Deposited material: 4 galls, 21-23.III.2001. Obtained insects: Hymenoptera.

Croton gnidiaceus Baill.: Globoid stem swelling (Fig. 39). Galling species: Cecidomyiidae. Period: November (CS). Deposited material: 1 gall, 21.XI.2001. Comments: first record of gall on this plant species.

Croton timandroides (Didr.) Müll. Arg.: Globoid stem swelling (Fig. 40). Galling species: Cecidomyiidae. Period: March (CM); May (CM). Deposited material: 3 galls (2 galls, 21-23.III.2001; 1 gall, 03.V.2002). 

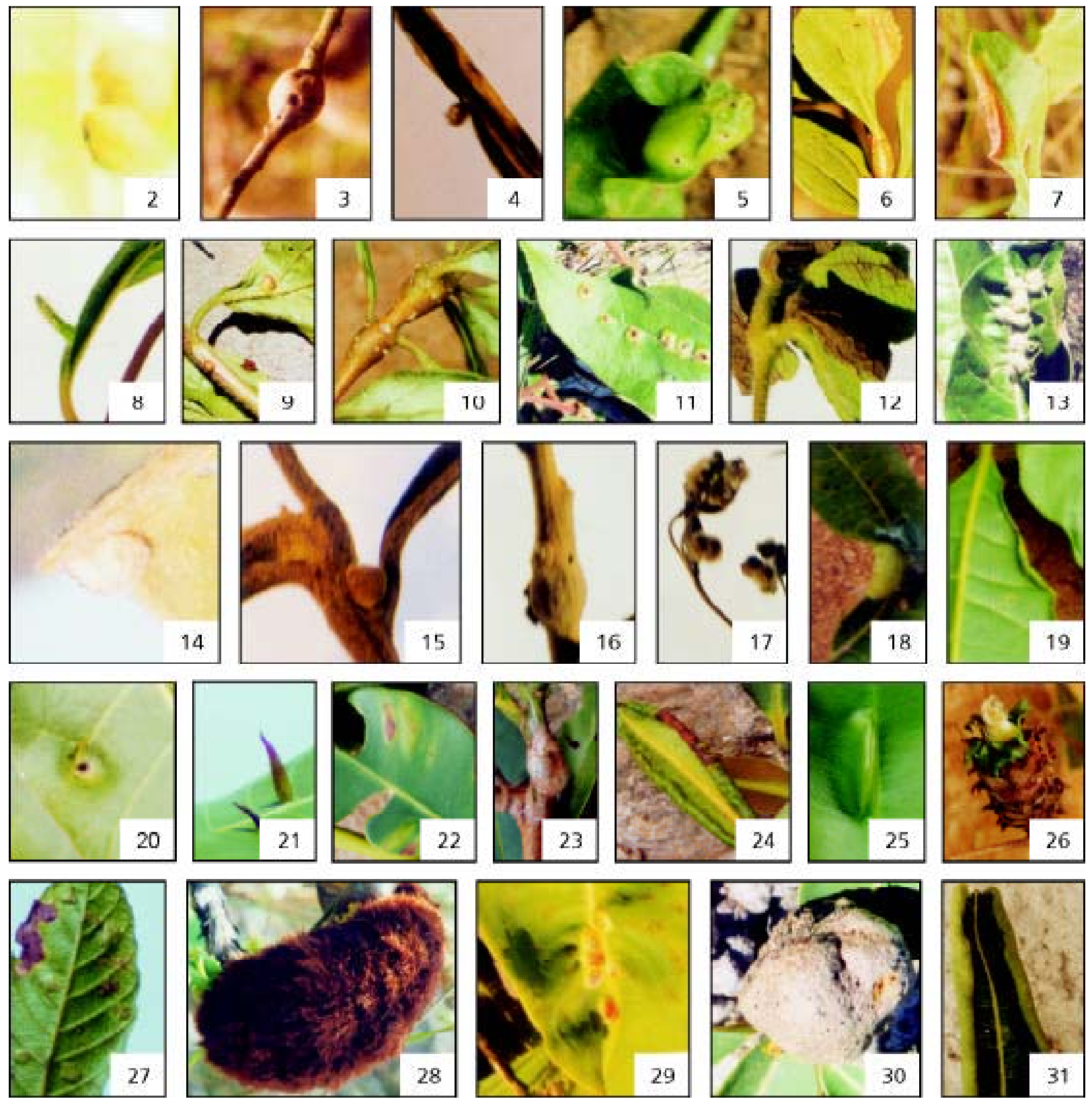

Figs. 2-31 - Insect galls of Serra de São José (Tiradentes, Minas Gerais). 2. Leaf gall on Anona crassiflora. 3. Stem gall on Aspilia duarteana. 4-5. Leaf galls on Baccharis microcephala: 4. Cylindrical gall; 5. Midvein swelling. 6-8. Galls on Baccharis reticularia: 6. Stem gall; 7. Marginal leaf roll; 8. Cylindrical outgrowths of the leaf. 9. Leaf or stem galls on Baccharis serrulata. 10. Stem gall on Mikania lindbergii. 11. Leaf galls on Mikania micrantha. 12. Stem gall on Mikania sessilifolia. 13. Leaf galls on Vannilosmopsis erythropapa. 14. Leaf gall on Vernonia crotonoides. 15. Bud or leaf gall on Vernonia obscura. 16. Stem gall on Vernonia sp. 17. Inflorescence gall on Cordia sp. 18-21. Galls on Protium heptaphyllum: 18. Pouch gall on leaf margin; 19. Marginal leaf roll; 20. Openned leaf gall; 21. Conical leaf gall. 22-25. Galls on Calophyllum sp.: 22. Elliptical leaf gall; 23. Stem gall; 24. Marginal leaf roll; 25. Triangular leaf gall. 26-27. Galls on Davilla braziliana: 26. Bud gall; 27. Leaf gall. 28-29. Galls on Erythroxylum suberosum: 28. Hairy leaf gall; 29. Circular leaf gall. 30-31. Galls on Erythroxylum frangulifolium: 30. Bud gall; 31. Marginal leaf roll. 
First record of gall on this plant species. Comments: Gagné (1994) related fusiform stem swellings caused by cecidomyiids on Croton buxifolius and C. migrans. Distr.: RJ and MG, Brazil. Ref. Rübsaamen (1905), Tavares (1922), and Houard (1933). Tavares (1925) and Houard (1933; Fig. B, p. 188) described spheroid leaf galls with stellate hairs induced by Styracodiplosis cearensis on $C$. hemiargyneus. Distr.: Ceará, Brazil. Rübsaamen (1905) recorded galls on lower and upper leaf surface caused by cecidomyiid on Croton sp. Distr.: RJ, Brazil. Rübsaamen (1905) and Houard (1933) listed marginal leaf rolls induced by cecidomyiid on Croton sp. Distr.: RJ, Brazil. Tavares (1915) and Houard (1933, Fig. 441, p. 188) related blister galls caused by cecidomyiid on $C$. argentinus. Distr.: Argentina. Houard (1933) listed a Eriophyidae gall on $C$. floribundus. Distr.: Brazil. Ref.: Rübsaamen (1905).

\section{Fabaceae}

Andira sp.: Globoid stem swelling (Fig. 41). Galling species: Cecidomyiidae. Other associated insects: parasitoids - Eulophidae ( 2 females from diferent species). Period: August-September (CM). Deposited material: 4 galls, 31.VIII.2001. Comments: Houard (1933, Figs. 249-250a-b) related a similar gall on Andira sp.

Globoid leaf gall, yellowish, glabrous (Fig. 42). Galling species: Cecidomyiidae. Other associated insects: parasitoids - Hymenoptera. Period: September (CM). Deposited material: 24 galls, 28.IX.2001.

Sinuous leaf gall. Galling species: Cecidomyiidae. Period: January (CM). Deposited material: 4 galls, 30.I.2002. Comments: Tavares (1920) described this gall and the galling species - Andirodiplosis bahiensis. Houard (1933) illustrated the gall (Fig. 258-260, 1-m).

Tapered stem swelling (Fig. 43). Galling species: Curculionidae (Coleoptera). Period: August (CM). Deposited material: 3 galls (2 galls, 31.VIII.2001; 1 gall, 28.IX.2002).

Copaifera langsdorffii Desf.: Horn-shaped outgrowths of leaves (Fig. 44). Galling species: Cecidomyiidae. Period: May (CM); August (CM); October (CM); January (CM), April-May (CM). Deposited material: 50 galls (1 gall, 03.V.2001; 47 galls, 25.V.2002; 2 galls, 28.V.2002). Comments. Fernandes \& Martins (1985) illustrated this gall (p.
62, Fig. 10); Fernandes et al. (1988) recorded it for Belo Horizonte (MG).

Globose leaf gall, brown and hairy. Galling species: undetermined insect. Period: May (CM). Deposited material: 18 galls, 03.V.2001.

Stem swelling. Galling species: Cecidomyiidae. Period: May (CM), January (CM). Deposited material: 1 gall, 03.V.2001.

Globoid leaf gall, green and glabrous (Fig. 45). Galling species: undetermined insect. Period: May (CM). Deposited material: 2 galls, 03.V.2001.

Discoid leaf gall, yellow (Fig. 46). Galling species: Cecidomyiidae. Other associated insects: parasitoids - Hymenoptera; inquilinous - Coleoptera (1 adult, 28.V.2002). Period: April-May (CM). Deposited material: 28 galls (03 galls, 03.V.2001; 25 galls, 25.IV.2002).

Bud gall, spheroid, green, with rough surface (Fig. 47). Galling species: Lepidoptera. Period: May (CM), December (CM). Deposited material: 13 galls (6 galls, 03.V.2001; 3 galls, 04.V.2001; 1 gall, 24.X.2001; 2 galls, 27.XII. 2001; 1 gall, 28.V.2002).

Inga sp.: Midvein swelling (Fig. 48). Galling species: Cecidomyiidae. Other associated insects: parasitoids - Hymenoptera. Period: October (CM); January (CM). Deposited material: 4 galls (1 gall, 25.X.2001; 3 galls, 30.I.2002). Comments: Houard (1933) listed and illustrated a similar gall (Fig. 129b). Maia (2001) described a similar gall on Inga maritima induced by Neolasioptera sp. (Cecidomyiiidae).

Globose, hairy, yellow leaf gall (Fig. 49). Galling species: undetermined insect. Period: April (CM). Deposited material: 3 galls, 25.IV.2002.

Comments: Gagné (1994) listed several galls on Inga spp. caused by Neolasioptera spp.

Stryphnodendron adstringes (Aubl.) Benth: Globoid stem swelling (Fig. 50). Galling species: Cecidomyiidae. Period: March (CM); May (CM); September (BD). Deposited material: 7 galls, 2123.III.2002. Comments: first record of gall on this plant species.

Undetermined Fabaceae (sp. 1)

Pouch gall on leaves. Galling species: undetermined insect. Period: November (CM). Deposited material: 1 gall, 20.XI.2001.

Swelling of midvein basis. Galling species: Cecidomyiidae. Period: April (CM) 

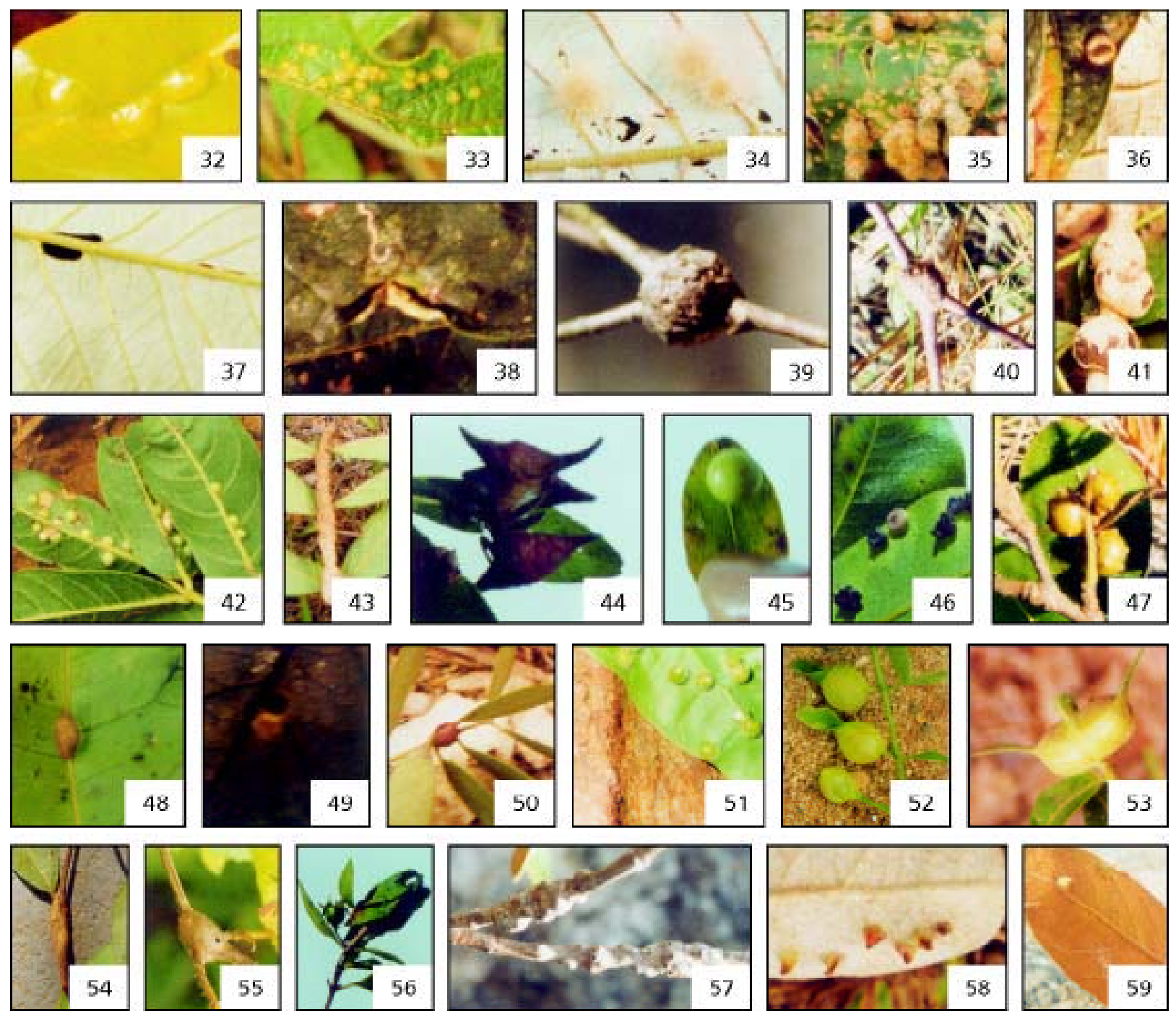

Figs. 32-59 - Insect galls of Serra de São José (Tiradentes, Minas Gerais). 32. Leaf gall on Erythroxylum sp. 33. Leaf galls on Croton antisyphiliticus. 34-38. Galls on Croton floribundus: 34. Spheroid leaf galls; 35. Ovoid leaf galls; 36. Discoidal leaf gall; 37. Midvein swelling; 38. Marginal leaf gall. 39. Stem swelling on Croton gnidiaceus. 40. Stem swelling on Croton timandroides. 41-43. Galls on Andira sp.: 41. Globoid stem swelling; 42. Globoid leaf gall; 43. Tapered stem swelling. 44-47. Galls on Copaifera langsdorffii: 44. Horn-shaped outhgrowths of leaves; 45. Globoid leaf gall; 46. Discoid leaf gall; 47. Bud gall. 48-49. Galls on Inga sp.: 48. Midvein swelling; 49. Leaf gall. 50. Stem gall on Stryphnodendron adstringens. 51. Leaf gall on Leguminosae sp.2. 52. Bud gall on Leguminosae sp.3. 53-54. Galls on Mimosaceae: 53. Bud gall; 54. Stem swelling. 55. Stem swelling on Hyptis sp. 56. Stem gall on Diplusodon virgatus. 57-58. Galls on Byrsonima variabilis: 57. Stem swelling; 58. Leaf gall. 59. Leaf gall on Byrsonima verbascifolia. 



Figs. 60-81 - Insect galls of Serra de São José (Tiradentes, Minas Gerais). 60. Stem galls on Byrsonima sp. 61-62. Galls on Leandra aurea: 61. Bud gall; 62. Leaf gall. 63. Vein swellings on Miconia sp.1. 64. Leaf galls on Miconia sp. 2. 65. Leaf and stem galls on Miconia sp. 3. 66-69. Galls on Miconia theaezans: 66. Rosette bud gall; 67. Spheroid bud gall; 68. Blister leaf gall; 69. Circular leaf gall. 70-71. Galls on Tibouchina candolleana: 70. Stem swelling; 71. Elliptical gall on leaves and stem. 72. Circular leaf gall on Rapanea andina. 73. Circular leaf gall on Campomanesia pubescens. 74. Stem swellings on Eugenia sp.1. 75. Globoid leaf gall on Eugenia sp.2. 76-77. Cylindrical outgrowths of leaves on Eugenia cf. ovalifolia: 76. Leaf upper surface view; 77. Leaf lower surface view. 78-81. Galls on Myrcia sp.: 78. Bud gall; 79. Midvein swelling; 80 . Leaf roll; 81. Marginal leaf roll. 

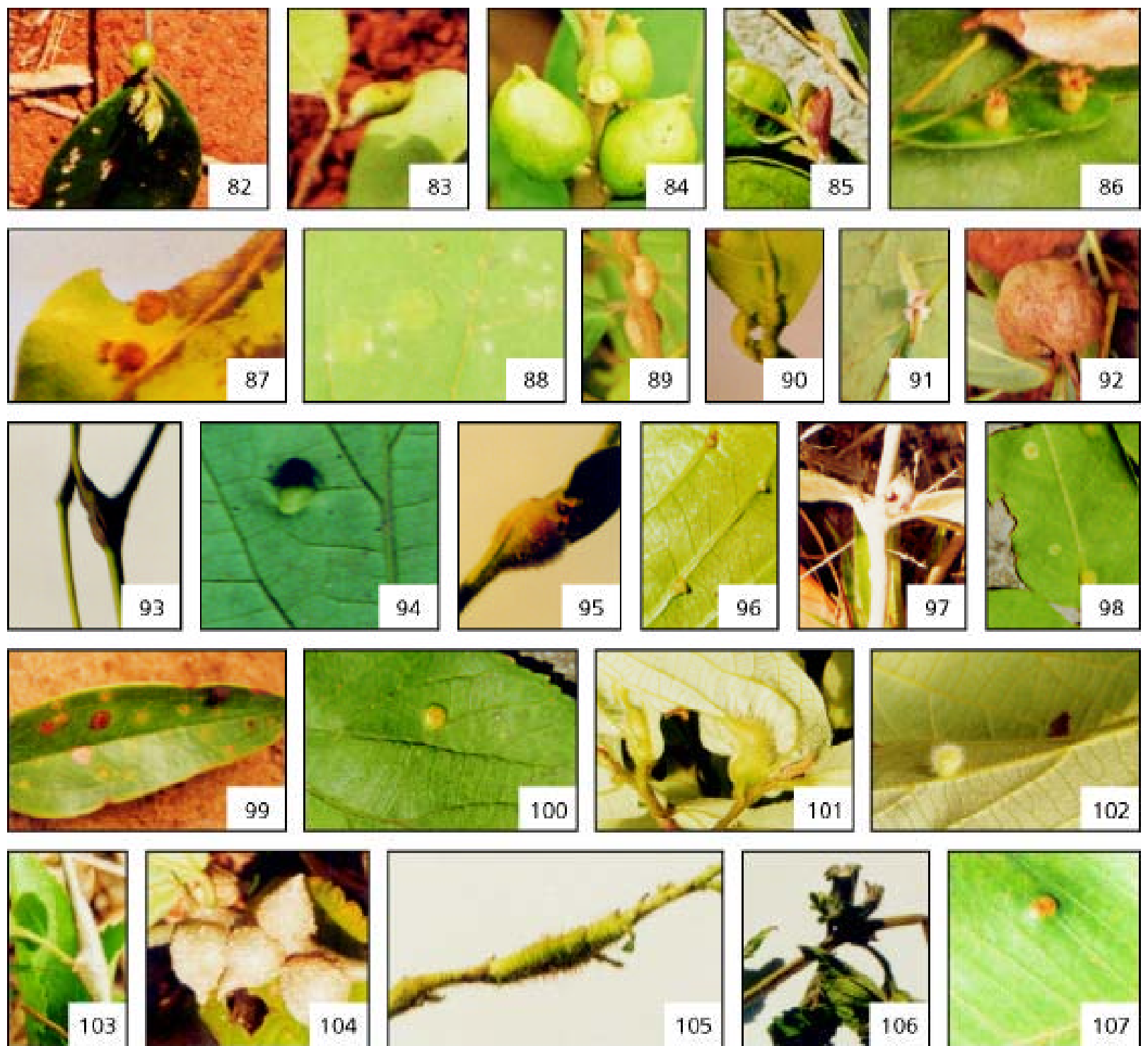

Figs. 82-107 - Insect galls of Serra de São José (Tiradentes, Minas Gerais). 82-83. Galls on Myrcia sp.: 82. Bud gall; 83. Fold leaf. 84. Bud gall on Psidium sp. 85. Fold leaf on Myrtaceae sp.1. 86-87. Galls on Myrtaceae sp.2: 86. Tubular leaf galls; 87. Globose leaf galls. 88-90. Galls on Guapira sp.: 88. Spot leaf gall; 89. Stem swelling; 90. Midvein swelling. 91. Leaf gall on Piper sp.1. 92. Stem swelling on Piper sp.2. 93-94. Galls on Piper sp.3: 93. Stem swelling; 94. Globoid vein swelling. 95. Stem swelling on Borreia cf. brachystemonoides. 96. Vein swelling on Palicourea rigida. 97. Bud gall on Sapicia brasiliensis. 98. Vein swelling and spot leaf gall on Paullinia sp. 99. Leaf gall on Smilax elastica. 100-101. Galls on Luehea cf divaricata: 100. Globoid leaf gall; 101. Midvein swelling. 102-104. Galls on Celtis glycycarpa: 102. Circular leaf gall; 103. Stem swelling; 104. Mamiliform outgrowths of leaves and stem. 105-106. Galls on Lantana lilacina: 105. Stem gall; 106. Tubular outgrowths of the leaves. 107. Leaf gall on Qualea parvifolia. 
Undetermined Fabaceae (sp. 2): Globose, hairy, green leaf gall (Fig. 51). Galling species: Cecidomyiidae. Period: May (CM).

Undetermined Fabaceae (sp. 3): Spherical bud gall, green (Fig. 52). Galling species: Cecidomyiidae. Mature larvae pupate into soil. Period: September (CM).

Undetermined Fabaceae (sp. 4)

Horn-shaped bud gall, yellowish (Fig. 53). Galling species: Curculionidae (Coleoptera, 1 adult, 27.XII.2001). Other associated insects: inquilinous Contarinia sp. (Cecidomyiidae); parasitoids Hymenoptera. Period: August (CM); NovemberDecember (CM and CS); January (CM); April-May (CM). Deposited material: 6 galls (2 galls, 31.VIII.2001; 3 galls, 20.XI.2001; 1 gall, 27.XII.2002).

Tapered stem swelling (Fig. 54). Galling species: Neolasioptera sp. (Cecidomyiidae). Other associated insects: parasitoids - Hymenoptera. Period: November (CM); December (CM and CS); January (CM); April-May (CM). Deposited material: 5 galls (2 galls, 21.XI.2001; 3 galls, 27.XII.2001).

Globoid leaf gall. Galling species: Cecidomyiidae. Period: November (CM).

\section{Lamiaceae}

Hyptis sp.: Stem swelling, hairy (Fig. 55). Galling species: Cecidomyiidae. Period: September-October (CM); April (CM). Comments: Möhn (1964) described a similar gall on Hyptis suaveolens and its galling species - Neolasioptera hyptis. Distr. El Salvador.

Globose hairy leaf gall. Galling species: Cecidomyiidae. Period: October (CM).

\section{Lauraceae}

Ocotea sp.: Opened leaf gall. Galling species: Coccoidea (Hemiptera, Sternorrhyncha). Period: April, 2002. Comments. Houard (1933) listed galls on Ocotea spp. and Monteiro et al. (1993) listed a similar gall induced by Sternorrhyncha on Ocotea notata.

\section{Lythraceae}

Diplusodon virgatus Pohl.: Apical stem swelling covered by leaves (Fig. 56). Galling species: Cecidomyiidae. Period: May (CM). Comments: first record of gall on this plant species.

\section{Malpighiaceae}

Byrsonima variabilis A. Juss.: Stem swelling, glabrous (Fig. 57). Galling species: undetermined insect. Period: September (BD); October-November (CM); January (CS and CM); April-May (CM). Deposited material: 20 gall, 29.IX.2001. Obtained insects: Sycophila sp.1 (Eurytomidae, 13 females, 1 male); Sycophila sp.2 (Eurytomidae, 5 females, 9 males); Eurytoma sp. (Eurytomidae, 2 females, 1 male); Eupelmidae sp. (1 male); Eulophidae sp. (2 females); Platygastridae sp. (1 male); Ichneumonidae sp. (1 female).

Conical leaf gall (Fig. 58). Galling species: Cecidomyiidae. Period: April (CM).

Comments: these are the first records of gall on Byrsonima variabilis.

Byrsonima verbascifolia A. Juss.: Conical leaf gall (Fig. 59). Galling species: Cecidomyiidae. Other associated insects: Eurytoma sp. (Eurytomidae, 1 female, 7 males); Eulophidae sp. (6 females, 5 males); Eupelmidae sp. (5 males); Dimeromicrus cecidomyiae (Torymidae, 1 male); Signiphoridae sp. (1 female); Psyllidae (1 adult). Period: March (BD); September (BD); October (CM); December (CM). Deposited material: 20 galls, 01.IX.2001. Comments: gall described by Tavares (1921), listed and illustrated by Houard (1933, Fig. G, p. 157-158), and related by Gonçalves-Alvim \& Fernandes (2001).

Byrsonima sp.: Stem swelling, hairy (Fig. 60). Galling species: undetermined insect. Period: November (BD). Deposited material: 1 gall, 21.XI.2001.

\section{Melastomataceae}

Leandra aurea (Cham.) Cogn.: Spherical bud gall, reddish, hairy (Fig. 61). Galling species: Lepidoptera (adults and immature specimens). Other associated insects: inquilinous - Anthonomus sp.n. (Coleoptera, Curculionidae; 9 adults, XI.2001); predators - Friebrigella sp. (Diptera, Chloropidae; 2 adults, 24.X.2001) and Lestodiplosis sp. (Diptera, Cecidomyiidae); parasitoids - Hymenoptera. Period: March (CM and BD); May, (CM); August (CM); September (BD); October (BD, CM and CS); November (CM); December-January (CM); April-May (CM). Deposited material: 4 galls, 21-23.III.2001. 
Spherical reddish leaf gall, hairy (Fig. 62). Galling species: Lepidoptera (4 adults, 27.XII.2001). Other associated insects: parasitoids - Hymenoptera. Period: May (CM); October (CM); December (CM); January (CM); May (CM). Deposited material: 6 galls, 21-23.III.2001.

Comments: Houard (1933) described and illustrated Eriophyidae galls on Leandra sp. (p. 291). These are the first records of gall on Leandra aurea.

Miconia sp.1: Tubular hairy leaf gall, green. Galling species: undetermined insect. Period: December (CM); May (CM). Deposited material: 5 galls, 28.XII.2001.

Vein swelling (Fig. 63). Galling species: Cecidomyiidae. Period: December (CM); May (CM).

Miconia sp.2: Globose, reddish and hairy gall on leaves and stem (Fig. 64). Galling species: Lepidoptera. Period: April. Deposited material: 2 leaf galls (24.IV.2002), 8 stem galls (24.IV.2002).

Miconia sp.3: Spheroid, hairy, reddish gall on leaves and stem (Fig. 65). Galling species: Cecidomyiidae. Other associated insects: parasitoids Hymenoptera. Period: November (CM); January (CM); May (CM). Deposited material: 8 galls, 24.IV.2002.

Miconia theaezans (Bonpl.) Cogn.: Rosette bud gall (Fig. 66). Galling species: Lepidoptera. Period: October (CM); January (CS and CM); April (CM); May (CS and CM). Deposited material: 10 galls (8 galls, 22.III.2001; 2 galls, 29.IX.2001).

Tubular bud gall. Galling species: Lepidoptera. Period: April-May (CM). Deposited material: 6 galls, 28.V.2002.

Spheroid bud gall (Fig. 67). Galling species: Cecidomyiidae. Other associated insects: parasitoids Hymenoptera. Period: April-May (CM). Deposited material: 6 galls, 25.IV.2002.

Blister leaf gall (Fig. 68). Galling species: Cecidomyiidae. Period: October (CM). Deposited material: 17 galls, 25.X.2001.

Circular leaf gall (Fig. 69). Galling species: Cecidomyiidae. Period: March (CM). Deposited material: 15 galls, 22.III.2001.

Comments: Houard (1933, p. 293-302) described several galls on Miconia spp. Gagné (1994) listed a tapered stem swelling caused by Cecidomyiidae. Rübsaamen (1907), Tavares (1925),
Houard (1933) described complex leaf galls induced by cecidomyiids. This is the first record of gall on this plant species.

Tibouchina candolleana (DC.) Cogn.: Vein swelling. Galling species: Cecidomyiidae. Other associated insects: parasitoids - Hymenoptera. Period: September-November (CM); December (CM and CS); January (CM); April, (CS and CM); May (CM). Deposited material: 12 galls (10 galls, 31.VIII.2001; 1 gall, 25.X.2001; 1 gall, 29.I.2002).

Stem swelling (Fig. 70). Galling species: Lepidoptera. Period: August (CM), October (CM). Deposited material: 6 galls (2 galls, 31.VIII.2001; 4 galls, 24.X.2001).

Elliptical gall on leaves or stem, hairy (Fig. 71). Galling species: Lepidoptera. Period: May, August, October (CM).

Comments: Rübsaamen (1908) described a vein gall on T. granulosa (Distr. RJ, Brazil); Tavares (1917) described two different leaf galls on Tibouchina sp., one spherical, hairy, and induced by Rochadiplosis tibouchinae, and the other a blister gall caused by an undetermined Cecidomyiidae. Houard (1933) listed both galls. This is the first record of gall on Tibouchina candolleana.

\section{Myrsinaceae}

Rapanea andina Mez.: Circular leaf gall (Fig. 72). Galling species: Cecidomyiidae. Other associated insects: parasitoids - Hymenoptera. Period: March (CS); May (CM); October (CM); December (CM). Deposited material: 10 galls (3 galls, 21-23.III.2001; 7 galls, 01.IX.2001). Comments: Maia (2001) recorded a similar gall induced by Cecidomyiidae on Rapanea parvifolia for the restinga of Barra de Maricá (Maricá, RJ, Brazil).

Stem swelling. Galling species: Lepidoptera. Period: October (CM). Deposited material: 1 gall, 24.X.2001.

Comments: first records of gall on Rapanea andina.

\section{Myrtaceae}

Campomanesia pubescens (DC.) O. Berg.: Circular leaf gall, yellowish (Fig. 73). Galling species: Cecidomyiidae. Period: May (CM); October (CM). Deposited material: 16 galls, 03.V.2001. Comments: first record on this plant species. 
Eugenia sp.1: Stem swelling (Fig. 74). Gall maker: Cecidomyiidae. Period: May (CM); September (CM).

Eugenia sp.2: Globoid leaf gall, brown, hairy (Fig. 75). Galling species: Cecidomyiidae. Period: May (CM). Deposited material: 35 galls, 03.V.2001.

Eugenia cfr. ovalifolia Camb.: Cylindrical outgrowths of leaves (Figs. 76-77). Galling species: Stephomyia sp. (Cecidomyiidae). Other associated insects: parasitoids - Hymenoptera. Period: September (CM). Deposited material: 65 galls, 01.IX.2001. Comments: Tavares (1921) described a similar gall and its galling species - Stephomyia clavata (as Oxasphondylia) on an undetermined species of Myrtaceae. Houard (1993) illustrated this gall (p. 276, Fig. e); Fernandes \& Martins (1985) recorded the occurrence of this gall on Eugenia ovalifolia in Belo Horizonte (MG, Brazil).

Myrcia sp.: Bud gall (Fig. 78). Galling species: Cecidomyiidae (probably Myrciamyia sp.). Other associated insects: Hymenoptera - inquilinous, modifies the appearance of the gall. Period: August, (CM); September (BD, CS and CM); October (CM); November (CM and CS); April, 2002 (CM). Deposited material: 7 modified galls, 31.VIII.2001. Comments: Maia (2001) described a similar gall induced by Myrciamyia maricensis on Myrcia ovata and also modified by an inquilinous Hymenoptera in the restinga of Barra de Maricá (Maricá, RJ, Brazil).

Midvein swelling (Fig. 79). Galling species: Neolasioptera sp. (Cecidomyiidae). Period: August (CM) Deposited material: 1 gall, 31.VIII.2001. Comments: Maia (2001) described a similar gall also induced by Neolasioptera on Myrcia ovata in the restinga of Barra de Maricá (Maricá, RJ, Brazil).

Leaf roll (Fig. 80). Galling species: Thrips (Thysanoptera, adults and nymphs). Other associated insects: Hymenoptera. Period: November (CM and CS); December-January (CM). Deposited material: 1 gall, 30.I.2002.

Marginal leaf roll (Fig. 81). Galling species: Cecidomyiidae. Other associated insects: parasitoids Hymenoptera. Period: September (BD and CM); October (CM); December (CM and CS); January (CM); April-May (CM). Deposited material: 10 galls, 01.IX.2001.
Apical bud gall, green with a terminal pointed projection (Fig. 82). Galling species: undetermined insect. Period: September (CM). Deposited material: 1 gall, 28.IX.2001.

Leaf fold along midvein (Fig. 83). Galling species: Hemiptera (Sternorrhyncha). Period: September (BD and CM); November-December(CM); April-May (CM). Deposited material: 4 galls, 31.VIII.2001.

Comments: Fernandes et al. (1988) recorded several galls on Myrcia itambensis in Belo Horizonte (MG, Brazil), none of them similar in shape.

Myrciaria tenella (DC.) O. Berg.: Bivalve bud gall. Galling species: Myrciariamyia sp.n. (Cecidomyiidae). Other associated insects: parasitoids - Hymenoptera. Period: October-December (CM). Deposited material: 4 galls, 21.XI.2001. Comments: Maia (2001) described a similar gall on Myrciaria floribunda induced by Myrciariamyia bivalva in the restinga of Barra de Maricá (Maricá, RJ, Brazil).

Leaf fold along midvein. Galling species: Hemiptera (Sternorrhyncha). Other associated insects: Muscomorpha (2 larvae, 30.I.2002) and Hymenoptera. Period: October (CM); December (CM). Deposited material: 13 galls (11 galls, 21.XI.2001; 2 galls, 30.I.2002).

Comments: first records of gall on Myrciaria tenella.

Psidium sp.: Globoid suculent bud gall (Fig. 84). Galling species: Cecidomyiidae. Period: August (CS); September (CS); November (CS).

Comments: Houard (1933) listed several galls on Psidium sp., none of them similar in shape.

Undetermined Myrtaceae (sp.1): Cylindrical leaf gall. Galling species: Cecidomyiidae. Period: August (CM). Deposited material: 1 gall on 31.VIII.2001.

Leaf fold along midvein (Fig. 85). Galling species: Hemiptera (Sternorrhyncha). Period: August (CM). Deposited material: 1 gall, 31.VIII.2001.

Undetermined Myrtaceae (sp.2): Tubular leaf gall, green, with small apical projections (Fig. 86). Galling species: undetermined insect. Period: August (CS); September (CM); April (CS). Deposited material: 21 galls (16 galls, 31.VIII.2001; 5 galls, 24.IV.2002). Obtained insects: Hymenoptera. 
Globose leaf gall, hairy (Fig. 87). Galling species: Cecidomyiidae. Period: August (CS). Deposited material: 3 galls, 31.VIII.2001.

Midvein basis swelling. Galling species: Cecidomyiidae. Period: August (CS). Deposited material: 1 gall, 31.VIII.2001.

\section{Nyctaginaceae}

Guapira sp.: Spot leaf gall (Fig. 88). Galling species: Lopesiini (Cecidomyiidae). Other associated insects: parasitoids - Hymenoptera. Period: September (CS); November (CM); December (CM and CS); January (CS and CM); April-May (CM). Deposited material: 10 galls (5 galls, 28.IX.2001; 5 galls, 20.XI.2001).

Stem swelling (Fig. 89). Galling species: Asphondyliini (Cecidomyiidae). Other associated insects: parasitoids - Hymenoptera. Period: September (CS); October (CM); November-January (CS and CM); May (CM). Deposited material: 3 galls, 28.IX.2001.

Midvein or petiole swelling (Fig. 90). Galling species: Cecidomyiidae. Period: December (CM); April (CM).

Comments: Maia (2001) recorded five kinds of Cecidomyiidae galls on Guapira opposita for the restinga of Barra de Maricá (Maricá, RJ), all induced by species of Asphondyliini. This is the first record of a non-Asphondyliini galling species on Guapira spp.

\section{Piperaceae}

Piper sp.1: Conical outgrowths of leaves and stem (Fig. 91). Galling species: Asphondyliini (probably a new genus and species). Other associated insects: parasitoids - Hymenoptera; inquilinous Lepidoptera (immature specimen, 21.XI.2001). Period: August-January (CS). Deposited material: 3 galls (2 galls, 31.VIII.2001; 1 gall, 21.IX.2001). Comments: Rübsaamen (1908) described a similar gall for Rio de Janeiro (Brazil).

Piper sp.2: Globoid stem swelling (Fig. 92). Galling species: Zalepidota $\mathrm{sp}$. (Cecidomyiidae). Period: August-September (CS); October (CM); April-May (CM). Deposited material: 6 galls (4 galls, 31.VIII.2001; 2 gals, 25.X.2001). Comments: Tavares $(1909,1925)$ described similar galls induced by Zalepidota piperis and Z. tavaresi on Piper sp.
Piper sp.3: Tapered stem swelling (Fig. 93). Galling species: Cecidomyiidae. Period: January (CS); May (CS). Deposited material: 2 galls, 27.V.2002.

Globoid vein swelling (Fig. 94). Galling species: Cecidomyiidae. Other associated insects: parasitoids Hymenoptera. Period: April (CS); May (CM).

Globoid bud gall. Galling species: Cecidomyiidae. Period: April (CS).

Comments: Rübsaamen (1908) and Möhn (1960) recorded stem swellings on undetermined Piper for Rio de Janeiro (Brazil) and El Salvador, respectively.

\section{Rubiaceae}

Borreria cfr. brachystemonoides Cham. \& Schltdl.: Stem swelling, hairy (Fig. 95). Galling species: Cecidomyiidae. Other associated insects: parasitoids - Encyrtidae sp. (2 adults). Period: May (CS). Deposited material: 01 gall, 27.V.2002. Comments: Houard (1933) described a similar gall of Cecidomyiidae on Borreria sp. (p. 388).

Palicourea rigida Kunth: Triangular vein swelling (Fig. 96). Galling species: Cecidomyiidae. Period: November (CM). Deposited material: 6 galls, 21.IX.2001. Comments: Gagné (1994) listed two Cecidomyiidae gall on Palicourea sp. for Costa Rica (none of them similar in shape). This is the first record of gall on Palicourea rigida.

Sapicia brasiliensis Wernhm: Bud gall, glabrous (Fig. 97). Galling species: Cecidomyiidae. Period: May (CM). Comments: Gonçalves-Alvim \& Fernandes (2001) reported the same gall on the same host plant species for Três Marias (MG, Brazil).

\section{Sapindaceae}

Paullinia sp.: Vein swelling (Fig. 98). Galling species: Cecidomyiidae. Other associated insects: parasitoids - Hymenoptera. Period: January (CS and $\mathrm{CM})$; April (CS and CM); May (CM). Deposited material: 8 galls (1 gall, 30.I.2002; 7 galls, 25.IV.2002).

Stem swelling. Galling species: Cecidomyiidae. Other associated insects: parasitoids - Hymenoptera. Period: January (CM); April (CS). Deposited material: 3 galls, 25.IV.2002.

Spot leaf gall (Fig. 98). Galling species: Cecidomyiidae. Period: April, May (CM).

Comments: Rübsaamen (1908) described a spheroid gall on Paullinia sp. in Brazil (Amazo- 
nas). Maia (2001) related a stem swelling on $P$. weinmanniaefolia for Rio de Janeiro (Brazil). Gagné (1994) recorded a rolled young leaf on $P$. weinmanniaefolia in Rio de Janeiro (Brazil).

\section{Scrophulariaceae}

Buchnera rosea Kunth.: Stem swelling. Galling species: Cecidomyiidae. Period: November (CS). Comments: first record of gall on this plant species.

\section{Smilacaceae}

Smilax elastica Griseb: Spot leaf gall (Fig. 99). Galling species: probably Smilasioptera candelariae (Cecidomyiidae). Period: August (CS); September (BD and CM); December (CM); January (CM); April (CS and CM). Deposited material: 28 galls (16 galls, 31.VIII.2001; 12 galls, 01.X.2001). Comments: Maia (2001) recorded a similar gall on Smilax rufescens for the restinga of Barra de Maricá, Maricá, RJ, Brazil).

\section{Tiliaceae}

Luehea $\mathrm{cf}$ divaricata Mart.: Globoid, hairy, yellowish leaf gall (Fig. 100). Galling species: Cecidomyiidae. Period: October (CM); December (CS); April (CS). Deposited material: 7 galls, 24.X.2001.

Comments: Gagné (1994) listed this gall for Minas Gerais (Brazil) and presented an illustration (Fig. 339, p. 304).

Midvein swelling (Fig. 101). Galling species: Cecidomyiidae. Period: August (CS); October (CM); December (CS). Deposited material: 6 galls, 30.I.2002.

Comments: Gagné (1994) listed this gall for Minas Gerais (Brazil) and presented an illustration (Fig. 339, p. 304).

Circular leaf gall. Galling species: Cecidomyiidae. Period: November-January (CS). Deposited material: 20 galls, 24.X.2001.

\section{Ulmaceae}

Celtis glycycarpa Mart. ex Miq.: Circular leaf gall, yellow (Fig. 102). Galling species: Cecidomyiidae. Period: May (CS).

Tapered stem swelling (Fig. 103). Galling species: Cecidomyiidae. Period: May (CS). Deposited material: 2 galls, 25.IV.2002.
Mamiliform outgrowths of leaves and stem (Fig. 104). Galling species: Neolasioptera sp. (Cecidomyiidae). Other associated insects: parasitoids - Hymenoptera. Period: August (CS); October (CM); December-January (CS); April-May (CS). Deposited material: 5 galls (1 gall, 31.VIII.2001; 4 galls, 27.XII.2001). Comments: Fernandes \& Martins (1985) illustrated a similar gall on an undetermined Ulmaceae (p. 63, Fig. 12).

\section{Umbelliferae}

Eryngium sp.: Flower bud gall. Galling species: Cecidomyiidae. Period: April (CS). Deposited material: 14 galls, 29.I.2002. Comments: first record of gall on Eryngium spp.

\section{Verbenaceae}

Lantana lilacina Desf.: Tapered stem gall, hairy (Fig. 105). Galling species: Neolasioptera sp. (Cecidomyiidae). Other associated insects: parasitoids - Hymenoptera. Period: September (CM and CS); November-December (CM). Deposited material: 9 galls (4 galls, 01.IX.2001; 5 galls, 28.IX.2001). Comments: Houard (1933) listed a similar gall induced by an undetermined Cecidomyiidae (p. 347, Fig. 803-804d-e).

Tubular outgrowths of the leaves (Fig. 106). Galling species: Cecidomyiidae. Period: May (CS). Deposited material: 8 galls, 27.V.2002. Obtained insects: Hymenoptera. Comments: Houard (1933) reported and illustrated the same gall (p. 347, Fig. 808-810k-m).

\section{Vochysiaceae}

Qualea parvifolia Mart.: Circular leaf gall. Galling species: Cecidomyiidae. Other associated insects: parasitoids - Hymenoptera. Period: May (CM). Deposited material: 14 galls, 03.V.2001. Comments: Gonçalves-Alvim \& Fernandes (2001) described and illustrated this gall (p. 92, Fig. 299).

\section{Winteraceae}

Drimys brasiliensis Miers.: Globoid bud gall, brown (Fig. 107). Galling species: Eulophidae (Hymenoptera). Period: March, (CS). Deposited material: 1 gall, 21-23.III.2001. Comments: Houard (1933) listed an insect gall on this plant genus. 


\section{DISCUSSION}

The majority of the galls from Serra de São José were induced by Cecidomyiidae (76,5\%). As the Cecidomyiidae cause about $70 \%$ of all described galls in the world, these results were expected. Gonçalves-Alvim \& Fernandes (2001) surveyed the cerrado vegetation of Três Marias (Minas Gerais) and showed similar results.

There was a predominance of galls on Fabaceae (=Leguminosae), Myrtaceae, and Asteraceae. Houard (1933) has already designated the Fabaceae as the family with the greatest richness of insect galls in Central and South America. Further, Gonçalves-Alvim \& Fernandes (op. cit.) indicated the predominance of galls on Fabaceae, Myrtaceae, and Asteraceae for cerrado vegetation.

Most galls were observed on leaves (65\%), a world pattern noted by Mani (1964), and corroborated by Maia (2001) for restinga areas and GonçalvesAlvim \& Fernandes (2001) for cerrado vegetation. The second most attacked plant organ was the stem. Similar results were presented by Gonçalves-Alvim \& Fernandes (op. cit.).

According to Fernandes \& Price (1988) and Lara \& Fernandes (1996), rupestrian fields and the cerrado vegetation of southeastern Brazil comprise the hottest spot for richness of galling insect species. Gonçalves-Alvim \& Fernandes (2001) recorded 92 spp of galling insects in Três Marias; 136 are recorded herein. These results corroborate the trend.

In comparing the host plant species and the insect galls from Serra de São José and Três Marias, we noted that these areas show a weak similarity. Only three kinds of galls occurred in both areas: spherical leaf gall, orangish green, hairy on Protium heptaphyllum; conical leaf gall on Byrsonima verbascifolia; and spheroid leaf gall, brown, glabrous on Sapicia brasiliensis.

For associated fauna, there was a predominance of parasitoid species of Hymenoptera, specially of the families Eulophidae and Eurytomidae families. This corroborates the trend found by Maia (2001) and Fernandes et al. (1988), in which these two families are identified as the most important natural enemies of gall midges in the Neotropical region.
Acknowledgements - We are grateful to R. J. V. Alves (MNRJ) for identifying the host plants (except for Asteraceae species) and for reviewing the manuscript; J. N. de M. Machado (IBAMA, Tiradentes) for logistic support; R. L. Steves (UERJ) for Asteraceae identification; D. Medeiros (MNRJ) for field assistance; L. de Bruyn (Institute of Nature Conservation) for Chloropidae identification; M. Couri (MNRJ) for manuscript review; and to CNPq for financial support to VCM (150061/ 01-3) and GWF (52.17722/95-8).

\section{REFERENCES}

ALVES, R. J. V., 1992, The flora and vegetation of the Serra de São José in Minas Gerais, Brazil. Tropicaleaf Nature Publishers, 67p.

BRANDÃO, M., CARVALHO, P. G. S. \& JESUÉ, G., 1992, Guia ilustrado de plantas do cerrado de Minas Gerais. CEMIG, Minas Gerais, 78p.

FERNANDES, G. W. \& PRICE, P. W., 1988, Biogeographic gradients in galling species richness. Test of hypotheses. Oecologia, 76: 161-167.

FERNANDES, G. W., CARNEIRO, M. A. A., LARA, A. C. F., ALLAIN, L. R., ANDRADE, G. I., JULIÃO, G. R., REIS, T. R. \& SILVA, I. M., 1996, Galling insects on neotropical species of Baccharis (Asteraceae). Tropical Zoology, 9: 315-332.

FERNANDES, G. W. \& MARTINS, R. P., 1985, As galhas. Tumores de Plantas. Ciência Hoje, 4: 58-64.

FERNANDES, G. W., NETO, E. T. \& MARTINS, R. P., 1988, Ocorrência e caracterização de galhas entomógenas na vegetação do campus Pampulha da Universidade Federal de Minas Gerais. Revta Bras. Zool., 5: 11-29.

GAGNÉ, R. J., 1989, The plant-feeding gall midges of North America. Cornell University Pres, Ithaca, xi $+356 \mathrm{p} ., 4$ pls.

GAGNÉ, R. J., 1994, The gall midges of the Neotropical Region. University Pres, Ithaca, xiv $+352 \mathrm{p} ., 4$ pls.

GAGNÉ, R. J., ODA, R. A. M. \& MONTEIRO, R. F., 2001, The gall midges (Diptera: Cecidomyiidae) of Mikania glomerata (Asteraceae) in Southeastern Brazil. Proc. Entomol. Soc. Wash., 103(1): 110-134.

GONÇALVES-ALVIM, S. J. \& FERNANDES, G. W., 2001, Comunidades de insetos galhadores (Insecta) em diferentes fisionomias do cerrado em Minas Gerais, Brasil. Revta Bras. Zool., 18(Supl. 1): 289-306.

HOUARD, C., 1933, Les zoocécidies des plantes de l'Amérique du Sud et de l'Amérique Central. Hermann et Cie, Paris. $519 \mathrm{p}$.

LARA, A. C. F. \& FERNANDES, G. W., 1996, The highest diversity of galling species: Serra do Cipó, Brazil. Biodiversity Letters, 3: 111-114.

MAIA, V. C., 2001, The gall midges (Diptera, Cecidomyiidae) from three restingas of Rio de Janeiro State, Brazil. Revta Bras. Zool., 18(2): 583-629. 
MAIA, V. C., COURI, M. S., LAMAS, C. J. E., PAMPLONA, D. \& AIRES, C. C. de C., 2002, A survey of the Diptera fauna of Serra do Cipó (Minas Gerais, Brazil). Entomologist's Monthly Magazine, 138: 109-117.

MANI, M. S., 1964, Ecology of plant galls. Junk, The Hague. 434p.

MÖHN, E., 1959, Gallmücken (Diptera, Itonididae) aus El Salvador. 1. Teil. Senck. Biol., 40: 297-368.

MÖHN, E., 1960, Gallmücken (Diptera, Itonididae) aus El Salvador. 2. Teil. Senck. Biol., 41: 197-240.

MÖHN, E., 1964, Gallmücken (Diptera, Itonididae) aus El Salvador. 7. Teil, Lasiopteridi. Deutsche Entomologische Zeitschrift, 11: 47-143.

MONTEIRO, R. F., FERRAZ, F., MAIA, V. C. \& AZEVEDO, M. A. P. de, 1993, Galhas entomógenas em restingas: uma abordagem preliminar. III Simpósio de Ecossistemas da Costa Brasileira, 3, ACIESP, 87: 210-220.

RÜBSAAMEN, E. H., 1905, Beiträge zur Kenntnis aussereuropäischer Zoocecidien. II. Beitrag: Gallen aus Brasilien und Peru. Marcellia, 4: 65-85, 115-138.

RÜBSAAMEN, E. H., 1907, Beiträge zur Kenntnis aussereuropäischer Zoocecidien. III. Beitrag: Gallen aus Brasilien und Peru. Marcellia, 6: 110-173.

RÜBSAAMEN, E. H., 1908, Beiträge zur Kenntnis aussereuropäischer Zoocecidien. III. Beitrag [cont]: Gallen aus Brasilien und Peru. Marcellia, 7: 15-79.
TAVARES, J. S., 1909, Contributio prima ad cognitionem cecidologiae Braziliae. Brotéria, Série Zoológica, 8: 5-28, pls. I-VIII.

TAVARES, J. S., 1915, Cécidologie Argentine. Brotéria, Série Zoológica, 13: 88-126, pls. II-V.

TAVARES, J. S., 1917, As cecidias do Brazil que se criam nas plantas da família das Melastomataceae. Brotéria, Série Zoológica, 15: 18-49, pls. I-V.

TAVARES, J. S., 1920, Cecidologia brazileira. Cecidias que se criam em plantas das famílias das Leguminosae, Sapotaceae, Lauraceae, Myrtaceae, Punicaceae, Aurantiaceae, Malpighiaceae, Sapindaceae, Umbelliferae, Loranthaceae, Apocynaceae, Urticaceae, Salicaceae e Graminae. Brotéria, Série Zoológica, 18: 82-125, pl. III.

TAVARES, J. S., 1921, Cecidologia brazileira. Cecidias que se criam em plantas das famílias das Leguminosae, Sapotaceae, Lauraceae, Myrtaceae, Punicaceae, Aurantiaceae, Malpighiaceae, Sapindaceae, Umbelliferae, Loranthaceae, Apocynaceae, Urticaceae, Salicaceae e Graminae. Brotéria, Série Zoológica, 19: 76-112, pl. I.

TAVARES, J. S., 1922, Cecidologia brazileira. As restantes famílias. Brotéria, Série Zoológica, 20: 5-48c, pls. XI-XIX.

TAVARES, J. S., 1925, Nova contribuição para o conhecimento da cecidologia brasileira. Brotéria, Série Zoológica, 22: 5-55, pls. I-V. 